\title{
ABFAB. Attachment to the breast and family attitudes to breastfeeding. The effect of breastfeeding education in the middle of pregnancy on the initiation and duration of breastfeeding: a randomised controlled trial [ISRCTN2 I 556494]
}

\author{
Della Forster*1, Helen McLachlan², Judith Lumley ${ }^{1}$, Christine Beanland ${ }^{3}$, \\ Ulla Waldenström ${ }^{4}$, Heather Harris ${ }^{5}$, Diane Earl ${ }^{5}$ and Kaye Dyson ${ }^{5}$
}

\begin{abstract}
Address: ${ }^{1}$ Centre for the Study of Mothers' and Children's Health, La Trobe University, 251 Faraday St, Carlton, 3053, Australia, ${ }^{2}$ Clinical School of Midwifery and Neonatal Nursing Studies, School of Nursing, La Trobe University, 251 Faraday St, Carlton, 3053, Australia, ${ }^{3}$ School of Nursing, Deakin University, 221 Burwood Hwy, Burwood 3125, Australia, ${ }^{4}$ Karolinska Institutet, Stockholm, Sweden and ${ }^{5}$ The Royal Women's Hospital, 132 Grattan St, Carlton, 3053, Australia

Email: Della Forster* - D.Forster@latrobe.edu.au; Helen McLachlan - H.McLachlan@latrobe.edu.au; Judith Lumley - J.Lumley@latrobe.edu.au; Christine Beanland - cbeanland@nbv.org.au; Ulla Waldenström - ulla.waldenstrom@omv.ki.se; Heather Harris - sadiechild@yahoo.co.uk; Diane Earl - diane.earl@rwh.org.au; Kaye Dyson - kaye.dyson@rwh.org.au
\end{abstract}

* Corresponding author

Published: 29 August 2003

BMC Pregnancy and Childbirth 2003, 3:5
Received: 15 May 2003

Accepted: 29 August 2003

This article is available from: http://www.biomedcentral.com/I47I-2393/3/5

(c) 2003 Forster et al; licensee BioMed Central Ltd. This is an Open Access article: verbatim copying and redistribution of this article are permitted in all media for any purpose, provided this notice is preserved along with the article's original URL.

\begin{abstract}
Background: It has proven difficult to reach World Health Organization (WHO) recommendations that infants be exclusively breastfed from birth to six months of age $[1,2]$, yet there is limited knowledge about interventions that are effective in increasing breastfeeding initiation and duration. Particularly lacking is evidence about how to maintain breastfeeding rates in countries which already have a high initiation of breastfeeding. This study aims to determine whether mid-pregnancy breastfeeding education, with a focus on either attitudes to breastfeeding or on technical aspects of breastfeeding, has an effect on rates of breastfeeding initiation and duration. Secondary aims of the study are to: explore what factors might affect the duration of breastfeeding and evaluate the interventions from the participant and childbirth facilitator perspectives.

Methods/Design: A randomised controlled trial (RCT) design will be used. Women having their first baby, and planning to give birth as public patients at the Royal Women's Hospital (RWH), Melbourne, will be approached at 18-20 weeks of pregnancy and invited to participate in the study. Participants will be randomly allocated to a control group or one of two group interventions: a previously designed and trialled tool to teach practical aspects of breastfeeding or an exploration of family attitudes to breastfeeding. The latter was developed and piloted by the investigators in conjunction with the group facilitators, prior to trial commencement. The interventions are planned to take place at 20-25 weeks. Data will be collected by questionnaire at recruitment, at interview in hospital after the birth and by telephone interview six months later. Medical/obstetric outcomes will be obtained from the medical record. The sample size (972) was calculated to identify an increase in breastfeeding initiation from 75 to $85 \%$ and an increase from 40 to $50 \%$ in breastfeeding at six months.
\end{abstract}




\section{Background}

It is widely accepted that breast milk is the ideal infant food and superior to all forms of artificial feeding. In acknowledgement of the benefits of breastfeeding, the WHO recommends that all infants should be exclusively breastfed from birth to six months of age. [1-3] In Australia this has been recognised by health policy makers who support the WHO recommendations. [4-6]

Victorian breastfeeding statistics for 1994-95 show a dramatic decline from hospital discharge to six months after the birth. At discharge from hospital, $73.9 \%$ of new mothers are breastfeeding decreasing to $69.5 \%$ at two weeks, $53.9 \%$ at three months and $40 \%$ at six months. [7]

Both State and RWH (Melbourne) data suggest that at hospital discharge, breastfeeding rates are close to the Commonwealth target initiation rate of $75 \%$. [8] When the figures from the RWH are examined more closely, there is considerable difference by subgroup of women. The figures at discharge from hospital (fully breastfeeding or express breasted milk) at the time of the initial NHMRC grant application were greater than $90 \%$ for women delivering in the Family Birth Centre, $82 \%$ in the private postnatal units and $70 \%$ in the public units (Harris 1996, personal communication). An audit of 700 medical records was undertaken in 1998, excluding women who would be ineligible for $A B F A B$, that is non-English speaking, multiparous women and those whose babies had died, and found that the discharge breastfeeding rate (fully breastfeeding or feeding expressed breast milk) of publicly booked women who met ABFAB inclusion criteria was $75.3 \%$. The RWH findings are consistent with reports that women who breastfeed are older, have more education, greater income and more social support. [911] This is typical of the women accessing the family birth centre [12] and private units within the hospital. Public patients, including those of non-English speaking background and lower socioeconomic means have lower average breastfeeding rates than the State and hospital mean. There seems to be good rationale for paying more attention to breastfeeding promotion in this group of women.

Although breastfeeding rates at discharge from hospital approximate Commonwealth target rates, [8] State figures reveal that breastfeeding rates decline rapidly after discharge. Anecdotal evidence suggests that this is also the case at the RWH (Harris 1996, personal communication) and is supported by other Australian [13] and international studies. $[14,15]$ A recent Australian study using the 1995 National Health Survey data estimated the proportion of children breastfed at discharge from hospital, three and six months of age during the period 1992-1995. The findings indicate that $82 \%$ of mothers are breastfeeding when they leave hospital, but by three months only $57 \%$ of infants are breastfed and by six months only $46 \%$ of infants are continuing to breastfeed, either exclusively or partially. [16] Breastfeeding duration rates are not reaching set goals as stated by Lund-Adams \& Heywood [17] and are unlikely to meet those targets by the year 2000 . This highlights the need for action to support the promotion of continued breastfeeding.

Several studies have identified maternal characteristics that are associated with increased breastfeeding duration. These factors include attitudes to breastfeeding such as prenatal intention to breastfeed, [10,18-20] maternal commitment to breastfeeding, [20,21] beliefs about breastfeeding, [22] planned duration of breastfeeding [9,21-23] and social learning. [22] Cox and Turnbull [24] identified that women who knew their own breastfeeding history and were themselves breastfed were more likely to breastfeed than women who were unaware.

Other factors were related to maternal confidence $[10,22,25]$ and social support. $[18,22]$ Some of these factors may be amenable to change through an antenatal intervention. [18,22]

Randomised controlled trials have been used to evaluate antenatal interventions that aimed to promote breastfeeding. $[11,26,27]$ Two of these studies $[11,26]$ utilised an antenatal intervention providing information about the health benefits and technical aspects of breastfeeding in either one antenatal class or a one on one education session. The interventions were evaluated in small randomised controlled trials, with samples drawn from specific ethnic and low-income groups identified as having low breastfeeding rates. The women participating in these trials had all indicated that they intended to breastfeed. Kistin et al., [11] concluded that additional prenatal support increased the breastfeeding initiation rate in lowincome urban black women, although the effect on breastfeeding duration was unclear. Wiles [26] measured maternal perception of the infant and perception of breastfeeding success and concluded that the intervention contributed to a successful breastfeeding experience. Neither of these studies could answer the question of whether an antenatal intervention could increase the duration of breastfeeding. However, an antenatal hands-on breastfeeding skills group provided to 59 primiparous Chilean women resulted in an increase in breastfeeding rates at six months postpartum to $94 \%$ compared with $67 \%$ in the control group, who attended an educational breastfeeding promotion session. [27] A recent Australian randomised controlled trial involving 70 primiparous women [28] has also reported an increase in breastfeeding duration following an antenatal intervention with a hands-on focus teaching technical breastfeeding skills. Redman et al [29] trialled a combination of antenatal and postnatal inter- 
ventions (using alternate allocation), in Newcastle (NSW), and found that although the women liked the interventions, and although most women received all components of the intervention, there was no difference in breastfeeding duration.

Other studies aiming at increasing women's knowledge of the benefits of breastfeeding did not increase breastfeeding duration. [30] Dix, [10] for example, reported that whilst most women agreed that breastfeeding is better for their baby than artificial feeding, this knowledge is not a strong incentive to breastfeed when compared with their own needs and responsibilities.

Researchers have also examined the effect of postpartum interventions on breastfeeding duration, although the findings of these studies are not consistent. Grossman et al., [9] and Schy et al., [23] did not have the statistical power to demonstrate a difference in duration of breastfeeding with in-hospital lactation counselling. Frank et al., [32] combined in-hospital lactation counselling with ongoing telephone counselling, and again found no effect, although this study too was underpowered. Jones \& West [31] reported an increase in breastfeeding at four weeks with a lactation visit and support in-hospital and at home. A trial is underway in Perth, Western Australia, comparing routine postnatal care with increased breastfeeding support during and after the postnatal hospital stay. [33]

A recent systematic review of the effectiveness of interventions to promote breastfeeding initiation concluded that breastfeeding initiation rates in developed countries were increased by "informal, small group...education delivered during the antenatal period" and "one to one health education", and that "peer support programs...delivered in the ante- and postnatal periods... [increased] both initiation and duration rates of breastfeeding among women on low incomes, and particularly among women who have expressed a wish to breastfeed". [[34], pv] The authors also found "packages of interventions" to be effective at increasing initiation and usually duration of breastfeeding as well, such as a "peer support program and/or a media campaign combined with structural changes to the health sector" (pvii). Many of the included randomised controlled trials had small numbers, and although the participants came from a diverse range of backgrounds, many of the women were of low income and specific ethnic groups. However, this review is of limited relevance when considering increasing breastfeeding duration in Australia as breastfeeding initiation is much higher here that in most of the countries where the included trials were carried out.
Some of the principles and practices of postnatal support for lactating women have been incorporated into modern maternity care, and may be effective. [35] Strategies such as the employment of lactation consultants, a motherbaby lactation day unit and 24-hour telephone counselling service are current practice at the RWH in Melbourne. Although these measures appear to have been effective in supporting women with their lactation, other interventions may be required to increase breastfeeding rates to the target level.

Our conclusion from reviewing the literature was that general information about breastfeeding does not seem to be as effective as caregivers would hope. There is some evidence that a hands-on approach, teaching technical breastfeeding skills is effective, and that attitudes to breastfeeding, as well as support, are important. There are few randomised controlled trials of reasonable size addressing these issues.

An antenatal intervention was designed for the trial to address attitudes to breastfeeding by providing an educational session based on group discussions and exploration of family attitudes towards breastfeeding. We are also testing the effect of a simple educational intervention focusing on the technical aspect of attachment to the breast using a previously designed tool. [28]

\section{Aims}

This study aims to determine whether breastfeeding education in the middle of pregnancy, with a focus on either attitudes to breastfeeding or on technical aspects of breastfeeding, has an effect on the breastfeeding rate at hospital discharge, and on the duration of breastfeeding.

Secondary aims of the study are:

- To explore what factors might affect the duration of breastfeeding;

- To evaluate the interventions from the participant and childbirth facilitator perspectives.

\section{Rationale}

Increasing breastfeeding duration in Australian women will ensure that more children in our community will be exposed to the benefits of optimal nutrition. The protective effects of breastfeeding against illness will result in healthier children and have the potential to deliver large cost savings to the health care industry. Breastfeeding is also cost saving for women. [36-40]

In spite of extensive evidence demonstrating the benefits of breastfeeding, there is limited knowledge about what interventions are effective in increasing the breastfeeding 
rate. Many studies on breastfeeding are published but very few are randomised controlled trials aimed at evaluating specific procedures. Whilst a recent systematic review of breastfeeding interventions [34] did show the benefit of some 'packages' of interventions, it is arguably of limited relevance when considering increasing breastfeeding duration in Australia and other countries where breastfeeding initiation rates are higher. This study will increase our knowledge about the effects of antenatal interventions focusing on attitudes and technical breastfeeding skills and will provide information that will guide the clinical practice of midwives, medical practitioners and other health professionals involved in breastfeeding education and antenatal care.

\section{Hypotheses}

1. That a class specifically focused on the practical aspects of breastfeeding, conducted in the middle of pregnancy, will increase both breastfeeding initiation rates and duration.

2. That two classes specifically focused on family and societal attitudes to breastfeeding, including a partner or significant other, conducted in the middle of pregnancy, will increase both breastfeeding initiation rates and duration.

\section{Outcome variables}

The principal outcomes of the study are the initiation and duration of breastfeeding in the first six months after the birth. Data collected for this outcome:

- Breastfeeding initiation;

- Breastfeeding status at six months after the birth;

- Discrete variables to measure exclusivity of breastfeeding;

- Attendance at intervention/s;

For the secondary aim of the study, the exploration of what factors might affect the duration of breastfeeding, the following variables will be collected:

- Background data such as age, education, marital status, ethnic background, religion, smoking status;

- The woman's antenatal (at recruitment) attitudes to breastfeeding and her perception of the attitudes of her partner (or significant other);

- The woman's postnatal (at discharge) attitudes towards breastfeeding and her perception of the attitudes of her partner (or significant other);
- Breastfeeding intention, including plans re length of breastfeeding duration;

- Parents' own breastfeeding history (if known)

- Attendance at regular childbirth classes in the later part of pregnancy;

- Model of antenatal care (obstetric, midwifery or general practitioner based models);

- Events such as use of obstetric analgesia, oxytocin and operative delivery;

- Characteristics of baby, such as sex and weight;

- Timing and outcome of first feed;

- Mother's confidence in ability to breastfeed (measured at about 2 days postpartum);

- Perceived knowledge of breastfeeding;

- Length of postnatal stay;

- Feeding at discharge from hospital;

- Postnatal support after discharge (domiciliary visits, support from partner; relatives and friends);

- Woman's perception of milk supply;

- Perception of advice received;

- Introduction of solids;

- Health of the baby;

- Maternal health issues after childbirth;

- Recommencement of employment;

- Maternal height and weight;

Other related outcomes are:

- Women's experiences of breastfeeding;

- Support by partner (or significant other), other family, members and friends;

- Breastfeeding complications, such as nipple pain, milk stasis and mastitis; 
- Help-seeking behaviour for problems related to breastfeeding;

- Attendance at regular childbirth classes;

- Reasons for ceasing breastfeeding, and related feelings;

- Reasons for not commencing breastfeeding

Outcome variables related to interventions:

- Attendance at classes;

- Evaluation of each class by participants;

- Retrospective evaluation of classes at in-hospital and sixmonth data collection points;

- Process evaluation related to adherence to intervention protocols;

- Qualitative interviews with childbirth facilitators

\section{Study design}

The study uses a randomised controlled trial design, consisting of three arms: a control group, and two intervention groups. The two interventions are different educational sessions, conducted in the middle of pregnancy. The design and analysis plan is for each intervention group to be compared with the control group, not for multiple cross-comparisons.

\section{Study population}

All eligible women booking to have a baby at the RWH (Melbourne) during the recruitment period will be approached to participate in the trial.

\section{Inclusion criteria}

Women booking as public patients

Having a first child

Less than 24 weeks pregnant at the time of recruitment, and not less than 16 weeks.

English speaking: able to speak, read and write in English

\section{Exclusion criteria}

Physical problems which prevent women from breastfeeding, such as certain types of breast surgery.

Choosing either private or Birth Centre care

\section{Sample size}

Initial power calculations were based on breastfeeding rates at discharge and at three and six months after the birth. Ideally these calculations should be based on data from primiparae in public care. However, statistics by parity and model of care were not available, except for the 1995 breastfeeding rates at discharge from the RWH (Harris 1996, personal communication) which were presented for public and private patients separately. These figures were $70 \%$ (full breastfeeding and expressed breast milk combined) in public patients and $82 \%$ in private patients.

It was assumed that the antenatal intervention would increase the breastfeeding rate at discharge to the level of private patients, from $70 \%$ to $82 \%$. In order to detect such a difference ( $80 \%$ power, $95 \%$ confidence) 214 women each group would be required.

In the state of Victoria (1994-1995), the breastfeeding rate decreased from $74 \%$ at discharge to $54 \%$ at three months and $40 \%$ at six months postpartum, corresponding to a reduction of $27 \%$ and $46 \%$ respectively. A corresponding decrease in the group of public patients at the RWH would result in a breastfeeding rate of $51 \%$ at three months and $38 \%$ at six months.

Two hundred and eight women per group would be required to identify with confidence an increase in the breastfeeding rate at three months from an estimated $51 \%$ in the control group to $65 \%$ (a balance between the $60 \%$ target of the Australian Government and $80 \%$ of the WHO) in the intervention groups. This sample would have the power to detect an increase at six months from estimated $38 \%$ in the control group to $52.1 \%$ in the intervention groups.

Taking into account an estimated loss to follow-up of $20 \%$ at the six-month telephone interviews, a final sample of 268 women per group $(214+20 \%)$ would be required for the study. By hypothesising that the two interventions have similar effects, the final sample was calculated as 804 women (268 multiplied by 3 ).

In 1999, sample size and power calculations were re-evaluated following a medical record audit, which demonstrated that the RWH breastfeeding initiation rate had increased to $75.3 \%$. The review also considered the fact that women who did not speak and write English fluently would not be included in the trial, and that this group had lower breastfeeding rates than Australian born women. The new sample size estimate was calculated with the aim of increasing the breastfeeding rate from $75 \%$ to $85 \%$, ( $95 \%$ confidence and $80 \%$ power) with the required sample size being 270 per group. Allowing for $20 \%$ loss to fol- 
low up, the sample size required for each group was 324 (ie total sample of 972).

\section{Recruitment}

Recruitment to the trial will take place in the Ultrasound Department when women attend for their mid-trimester scan. By recruiting women in weeks $18-20$, it is envisaged that there will be very few women recruited who subsequently have a miscarriage or an intra-uterine death. It is also the one place and appointment that almost all women booking at the hospital for birth attend, apart from the initial booking visit.

\section{Assessment of eligibility}

Research midwives will liaise with staff in the Ultrasound Department to obtain daily ultrasound lists, then as much as possible, cross check this list with a hospital database to assess parity. At this point the database will also be utilised to verify that the scan is a routine mid-trimester scan and not another type.

\section{Recruitment protocol}

Recruitment is scheduled to take place at the routine ultrasound examination, around the $18^{\text {th }}$ gestational week. It is thought that women might be more motivated to attend classes after 18 weeks gestation than before, and after having had a scan confirming the viability of the fetus. Piloting demonstrated that in practice women will need to be approached immediately prior to the ultrasound scan, as most women left the ultrasound department very quickly following their scan, and did not have time to talk to the research midwife. Partners are often present at the ultrasound examination, which may facilitate women's recruitment to the study. Recruitment is scheduled close to the intervention, which takes place as soon as possible after the scan.

Research midwives will follow the recruitment guide (see Additional file: 1: Appendix 1) after determining that a woman is potentially eligible. The research midwife will explain the study and ask for consent to participate in the trial.

\section{Participant information}

Women will be sent written information (a brochure) about the study when they ring to book into the hospital for their pregnancy care (for brochure wording see Additional file: 1: Appendix VIII). Another copy of the pamphlet will be given to the woman at the time of recruitment, and she will have the opportunity to think about the study, read the pamphlet, and then consent if she wishes.

\section{Informed consent}

Written consent will be obtained if the woman agrees to enter the study. This will be witnessed and signed by another person, as well as the research midwife (see Additional file: 1: Appendix II).

\section{Intervention allocation \\ Randomisation procedure}

When a woman agrees to enter the study she will be randomly allocated to one of the three trial arms.

A computerised system of biased urn randomisation [41] will be accessed by telephone to ascertain women's allocation to one of three groups. The research midwife will be asked to follow prompts on the telephone, including inputting the woman's hospital record number. A randomised allocation to one of the groups is then generated.

The woman will be informed of the randomisation outcome at that time, and if allocated to a class, can choose a date and time that suits her. A reminder letter will be sent a week prior to the class.

\section{Study participation \\ Baseline}

After consenting to participate in the study, and prior to randomisation, women will be asked to fill out a baseline questionnaire (not included in protocol), which includes demographic data, and asks about breastfeeding intentions.

\section{Interventions}

Women will be allocated to either a control arm, or one of two education interventions (described below in 4.6), aimed to take place in the middle of pregnancy, prior to commencement of standard childbirth education classes.

\section{Follow up}

Women will be visited by the research midwife while in hospital following the birth, allowing a minimum of 48 hours when possible. A structured interview with both closed and open-ended questions (not included in protocol) will be conducted, taking about 10 minutes. The protocol allows for telephone follow-up at home for this initial interview if women are discharged home prior to being contacted in hospital by the research midwife. The hospital records will be checked before approaching women after the birth, and in this way women who have experienced a fetal death, an early neonatal death, those who have a seriously ill baby or who are seriously ill themselves are not approached. Women whose baby is seriously ill at the time of the in-hospital interview (and therefore not approached) will be included in the sixmonth follow-up, subject to confirmation that her baby was discharged home from hospital in good health. 
The final interview is scheduled to take place when the baby is six months old. This structured interview with both closed and open-ended questions (not included in protocol) is conducted by telephone, and takes 10-15 minutes.

\section{Study completion}

Successful completion of the study occurs after the sixmonth telephone interview. If desired, women will be sent a summary of the results after completion of the analyses.

\section{Participant discontinuation}

Women can withdraw informed consent at any time. No further study follow up will occur if they do so.

\section{Interventions}

\section{Description of 'attach' intervention}

The first intervention ('Attach') focuses on breastfeeding skills and utilises the teaching aids developed by Rebecca Glover $[42,43]$ and trialled in a small Australian study. [28] Only one class is offered, between approximately 20 and 25 weeks gestation, and class size aimed to not exceed eight women. The classes are conducted by midwives or health educators with specific training in childbirth education. The session focuses entirely on the technical aspects of breastfeeding (see Additional file: 1: Appendix III). The technique of attachment of the baby to the breast is explained and demonstrated using dolls and knitted 'breasts'. Breastfeeding complications are discussed and advice given about how to manage breastfeeding problems. Partners are not included in this intervention.

\section{Description of 'family' intervention}

The second intervention ('Family') is focused on changing attitudes to breastfeeding. Two classes are offered between 20 and 25 weeks gestation (see Additional file: 1: Appendix IV). The number of participants per class should not exceed eight women plus their partners or significant others. This intervention was designed specifically for the trial, by the investigators, in conjunction with the class facilitators. The content of the first class includes information about the advantages of breastfeeding, as well as an exploration of the expectant parents' views and attitudes on breastfeeding and their perceptions of the views of their family and friends. Community attitudes are also explored. As a preparation for the next class each participant will be encouraged to interview her own mother and her partner's mother about how they fed them as babies, and about the mother's present attitudes to breastfeeding. The second class is a group discussion based on these interviews and participants' reflections, as well as a discussion of resources available for breastfeeding women and encouragement to develop a breastfeeding plan.
The difference in the number of classes offered in the two interventions was motivated by a belief that more time and participant involvement (interviewing mothers, group discussions) is required to influence attitudes to breastfeeding than to learn about technical skills, whereas a small previous study suggests that only one session about how to attach the baby to the breast can affect duration of breastfeeding [28]. A crucial part of the family attitudes intervention is that the woman and her partner or significant other explore the attitudes of their families and friends and then have the opportunity to reflect on these with other participants and the group leader.

The timing of the interventions, before 25 weeks gestation, was motivated by a widespread view among childbirth educators that women attending ordinary childbirth classes at the end of pregnancy (usually after 30 weeks gestation) are focused on the birth itself, and that they are less interested in events after the birth, such as breastfeeding. By conducting the classes earlier in pregnancy, we plan not to compete with the regular programs, and the expectant parents still have the opportunity to participate in such classes.

\section{Process evaluation}

A number of strategies are in place to monitor intervention uptake and assess adherence to protocols.

\section{Measures of intervention attendance}

Facilitators for each class will record class lists (see Additional file: 1: Appendix XI), and this information will be cross-referenced with the trial database to measure intervention attendance. Women will also be asked what classes they attended at the subsequent interview.

\section{Adherence to protocols}

Facilitators for each class will be required to complete an evaluation form at the completion of class including if and where the class deviated from the protocol (see Additional file: 1: Appendix IX).

\section{Facilitator meetings}

Each group of facilitators will meet together, with the research team, on a regular basis (approximately six monthly) to discuss classes, adherence to protocols, and to receive feedback on participants' evaluations. Facilitators will be involved in only one arm of the trial, and 'attach' and 'family' facilitators will meet separately.

\section{Intervention evaluation by participants}

The views of the women and their partners or support people regarding the classes will be evaluated at the completion of each class. A structured questionnaire including open-ended questions will be used for this (see Additional file: 1: Appendix X). 


\section{Evaluation of experiences of class facilitators}

Class facilitators will be interviewed at the completion of the trial, prior to publication of trial results, regarding their views and experiences of being part of the trial (interview schedule not included in protocol).

\section{Data}

Data collection

Data collection tools

These are described in 6.3.3, and are not included as part of this protocol.

\section{Data collection methods}

The background demographic data questionnaire will be filled in by the women themselves at time of recruitment.

The interview after the birth will be conducted by a research midwife, in person, while the woman is still in hospital. If the woman has been discharged, the interview will be conducted by telephone, after allowing a day or two for the woman to settle in to being at home with her new baby.

The six-month interview will be conducted by the research midwife, by telephone. In all cases, the telephone protocol (see 5.1 .3 below) will be adhered to.

An Access database [43] connected to the hospital database will automatically receive medical/obstetric information after each woman has her baby. The project coordinators will maintain and manage this database.

\section{Telephone protocol}

All telephone interviewing will be conducted using the appropriate questionnaire, and according to the telephone protocol. This protocol includes instructions on contact procedures and documentation, as well as guidelines for the circumstance of a woman being upset or distressed (see Additional file: 1: Appendix V).

\section{Data coding}

Data coding schedules have been devised for each questionnaire (not included in this protocol), as well as instructions on how to ask each question, for example, whether to prompt answers, so that all interviewers ask and code questions the same way.

Regular meetings between the research midwives will expand on this process, to ensure clarity and a consistent approach.

\section{Data management}

\section{Data security and storage}

Data will be stored in locked filing cabinets in locked rooms, and accessed only by project coordinators.
Computer files will be password protected, and will be accessed only by project coordinators. Data linking women's names and study ID will be kept on a separate database to women's questionnaires, which will be identified by study ID only.

\section{Data entry}

Quantitative data will be entered by a data-entry company, with double data entry.

Qualitative data entry (open-ended questions) will be undertaken by project coordinator/s.

Data cleaning

To be undertaken using a variety of approaches, using an Access database [43] and STATA statistical package. [44]

\section{Data analysis}

Data will be collected to meet the CONSORT guidelines [45] for reporting of randomised trials.

- The first stage of analysis will check the comparability of participants allocated to the three groups.

In relation to the trial hypotheses, each intervention group will be compared with the group of women allocated to standard care by intention to treat analysis, which will also adjust for any "cluster" effect within classes.

- The duration of breastfeeding (exclusive and partial) will be compared by survival analysis.

- Proportions of women breastfeeding at hospital discharge, three and six months will be compared by odds ratios and chi-square tests.

- Comparison of means will be undertaken using t-tests where data are normally distributed.

- Ranked or Likert type scales will be analysed using Mann-Whitney U tests, and/ or cumulative odds ratios.

- If there are any differences in the characteristics of women in the three groups which might be associated with the major outcomes, a supplementary multivariate analysis will be carried out.

Although the primary analysis will be an 'intention to treat' analysis, a secondary analysis will repeat the comparisons above taking into account the additional factor of actual class participation, adjusting for any 'cluster' effect within classes. 


\section{Study administration}

Personnel

Participating investigators

Professor Judith Lumley (Centre for the Study of Mothers' and Children's Health, La Trobe University)

Ms Della Forster (Centre for the Study of Mothers' and Children's Health, La Trobe University)

Dr Helen McLachlan (Clinical School of Midwifery and Neonatal Nursing Studies, School of Nursing, La Trobe University)

Dr Christine Beanland (Nurses Board of Victoria)

Professor Ulla Waldenström (Karolinska Insitutet, Stockholm)

Ms Heather Harris (Royal Women's Hospital)

Ms Kaye Dyson (Royal Women's Hospital)

Ms Diane Earl (Royal Women's Hospital)

Associate Investigator: Dr Lisa Amir (Centre for the Study of Mothers' and Children's Health, La Trobe University)

Project team

Judith Lumley

Della Forster

Helen McLachlan

Christine Beanland

Heather Harris

Kaye Dyson

Diane Earl

Project coordinator/s

Della Forster

Helen McLachlan

Research assistant/s

Julie Manley

Ruth Bergman

Jenni James

Sally von Bibra
Job descriptions of required personnel

POSITION DESCRIPTION: Project Coordinator/s (see Additional file: 1: Appendix VI)

POSITION DESCRIPTION: Research Midwife (see Additional file: 1: Appendix VI)

\section{Staff education}

Research midwives will be required to undertake three days of education. Topics will include the project overall, the data collection tools, the recruitment process and interview techniques. This process will include actual recruitment and interviews under supervision, then alone, with close contact with project coordinator/s (see Additional file: 1: Appendix VII).

\section{Staff meetings}

It is anticipated that the research project team will meet monthly throughout the project, and more often as necessary.

Timelines

The project, from the commencement of recruitment, is planned to take place within three years (see Additional file: 1: Appendix XII). This includes recruitment of ten women per week, requiring approximately 97 weeks for recruitment. Each woman will give birth and the have her first interview approximately 20 weeks after recruitment, then her second interview is planned for six months after the birth. Following recruitment of the final woman, it will take 46 weeks to finalise data collection. Some writing up of the project will be undertaken within the recruitment and data collection phases, with analysis and final writing up following the completion of data collection.

\section{Documents required}

Information sheet

An information brochure will be sent to all women when they ring and book into the hospital, and then given to them again at recruitment (see Additional file: 1: Appendix VIII). The format used is a leaflet with the trial logo.

\section{Consent form}

Written consent will obtained if the woman agrees to enter the study. This will be witnessed by another person, as well as the research midwife (see Additional file: 1: Appendix II).

Data collection forms

- Background demographic questionnaire

- Interview following birth questionnaire

- Six-month interview questionnaire 
- Access database [43] connected to hospital database to automatically receive medical/obstetric information after each woman had her baby

- Medical/obstetric data sheet for those who give birth elsewhere

- Summary interview for women missing first interview, but having six-month interview

- Daily recruitment figures form (see Additional file: 1 : Appendix XIII)

- Class evaluation forms 'attach' and 'family' (see Additional file: 1: Appendix X)

- Facilitator evaluation forms (see Additional file: 1: Appendix IX)

Coding schedules

- Background questionnaire

- Interview following birth questionnaire

- Six-month interview questionnaire

\section{Ethical considerations}

Ethics approval/s

Approval was received from:

Research and Ethics Committee, Royal Women's Hospital, project number $97 / 25$

Human Ethics Committee, La Trobe University, number $96 / 97$

\section{Informed written consent}

Written consent will be obtained if the woman agrees to enter the study. This will be witnessed by another person, as well as the research midwife (see Additional file: 1: Appendix II).

Risks/ inconveniences/benefits

It is not anticipated that this trial poses any risks to women who participate. It is possible that they will benefit from the intervention if they are allocated to one of the two intervention arms, but this is unknown. The inconvenience would be class attendance for those allocated to interventions, and the time taken for interviews for all women, however this will be explained to women at the time of recruitment.

\section{Freedom to withdraw at any time}

Women can withdraw consent to participate in the trial at any time. If a woman withdraws there will be no further contact or follow-up.

Interim analysis

This is not planned due to the long duration of follow-up. Even six months of recruitment would require up to 72 weeks of follow-up to have an adequate sample for interim analysis. This would be too late for an interim analysis to give findings to stop the trial early (if required), to minimise harm.

Background data will be analysed following completion of recruitment to ascertain homogeneity of the three groups.

\section{Limitations}

It is possible that the sample size may need to be larger than planned if there are large 'group' effects. It is unknown what the rate of attendance at the intervention will be, and this could also affect the appropriateness of the chosen sample size.

\section{Abbreviations}

ABFAB Attachment to the Breast and Family Attitudes to Breastfeeding

NHMRC National Health and Medical Research Council

RCT Randomised controlled trial

RWH Royal Women's Hospital (Melbourne)

WHO World Health Organization

\section{Funding}

Funding has been obtained from NHMRC and PhD scholarship from The Royal Women's Hospital (2002) and VicHealth (Victorian Health Promotion Foundation) (2003-2004).

\section{Declaration of competing interests}

None known

\section{Authors' contributions}

Ulla Waldenström (UW), Christine Beanland (CB), Heather Harris $(\mathrm{HH})$, Judith Lumley (JL), Diane Earl (DE), Della Forster (DF), Helen McLachlan (HMc), Kaye Dyson (KD)

Chief investigator/ overall trial responsibility: UW (Chief Investigator (A) from study conception until 1997, when took up the Foundation Chair of Caring Sciences, Karolinska Insitutet. Project supervision transferred in late 1998); 
CB (Chief Investigator (B) from study conception until late 1997); JL (Chief Investigator from late 1998).

Study conception: UW

Study design: UW, CB

Initial grant application: UW, CB, HH, DE

Initial protocol draft: UW, CB

Protocol revision: DF, $\mathrm{HMc}, \mathrm{JL}, \mathrm{CB}$

Questionnaire first drafts: UW

Questionnaire development, piloting and completion: DF, HMc, JL

Questionnaire review: $\mathrm{CB}, \mathrm{HH}, \mathrm{DE}, \mathrm{KD}$

Intervention conception: UW

Intervention development: JL, $\mathrm{CB}, \mathrm{DE}, \mathrm{HH}$

Intervention piloting and refinement: $\mathrm{CB}, \mathrm{KD}, \mathrm{DE}$

Education of facilitators: $\mathrm{CB}, \mathrm{KD}, \mathrm{DE}$

Medical record audit: $\mathrm{CB}, \mathrm{HH}$

Coordination and implementation of trial: DF, HMc, JL

Development of process evaluation: $\mathrm{CB}, \mathrm{DF}, \mathrm{HMc}$, JL

Ongoing review of trial processes: $\mathrm{DF}, \mathrm{HMc}, \mathrm{JL}, \mathrm{HH}, \mathrm{DE}$, KD

Development of data collection process: DF, HMc, UW

Data collection, analysis and management: DF, HMc

Drafted trial protocol manuscript: DF

All authors read and approved final manuscript.

\section{Additional material}

\section{Additional File 1}

Appendix I: Recruitment dialogue; Appendix II: Consent form Appendix III: "Attach" class protocol; Appendix IV: "Family" class 1 and 2 protocol; Appendix V: Telephone protocol; Appendix VI: Position descriptions; Appendix VII: Research midwife orientation program; Appendix VIII: Information brochure; Appendix IX: Facilitator evaluation forms; Appendix X: Class evaluation forms; Appendix XI: Class lists; Appendix XII: Trial timeline

Click here for file http://www.biomedcentral.com/content/supplementary/14712393-3-5-S1.doc]

\section{Acknowledgements}

- The childbirth educators at The Royal Women's Hospital who participated in the design of the family attitudes intervention.

- The Childbirth Education Department at The Royal Women's Hospital

- The student midwives from La Trobe University who participated in trialling the interventions

- The women who participated in trialling of questionnaires

- Roger Short for his assistance during the transitional period between Chief Investigators

- The National Health and Medical Research Council for trial funding

- The Royal Women's Hospital and VicHealth for PhD scholarship funding

\section{References}

I. Kramer MS and Kakuma R: Optimal duration of exclusive breastfeeding (Cochrane Review). The Cochrane Library 2002.

2. World Health Organization: The optimal duration of exclusive breastfeeding. Results of a WHO systematic review. 2001 [http://www.who.int/inf-pr200I/en/note200I-07].

3. World Health Organization: Breast-feeding the technical basis and recommendations for action. World Health Organization: Geneva 1993.

4. Better Health Commission: Looking Forward to Better Health Volume 2. Australian Government Publishing Service: Canberra 1986.

5. National Health and Medical Research Council: Nutrition Policy Statements. Department of Community Services and Health, National Health and Medical Research Council: Canberra 1990.

6. National Health and Medical Research Council: Dietary guidelines for Australians. Australian Government Publishing Service: Canberra 1992.

7. Department of Human Services Victoria: MCH 1994/95 Annual Data Summary. Statewide Breastfeeding Details. DHS 1995.

8. Nutbeam D, Wise M, Bauman A and Harris E: Goals and Targets for Australia's Health in the Year 2000 and Beyond. Australian Government Publishing Service: Canberra 1993.

9. Grossman LK, Harter C, Sachs L and Kay A: The effect of postpartum lactation counseling on the duration of breast-feeding in low-income women. Am J Dis Child 1990, I44(4):47|-474.

10. Dix DN: Why women decide not to breastfeed. Birth 1991, I 8(4):222-225.

II. Kistin N, Benton D, Rao S and Sullivan M: Breast-feeding rates among black urban low-income women: effect of prenatal education. Pediatrics 1990, 86(5):741-746.

12. Handfield B and Bell R: Do childbirth education classes influence decision making about labor and post-partum issues. Birth 1995, 22(3): $153-160$.

13. Hitchcock $N$ and Coy J: Infant feeding practices in Western Australia and Tasmania: a joint survey, 1984-1985. Med J Aust 1988, 148: 114-117.

14. Emery JL, Scholey S and Taylor EM: Decline in breastfeeding. Arch Dis Child 1990, 5:369-372.

15. Essex C. Smale $P$ and Geddis D: Breastfeeding rates in New Zealand in the first six months and the reasons for stopping. $N Z$ Med J 1995, 95:355-358.

16. Donath S, Amir LH and Rates of breastfeeding in Australia by State and socio-economic status: Evidence from the 1995 National Health Survey. J Paediatr Child Health 2000, 36: I64-168.

17. Lund-Adams M and Heywood P: Australian breastfeeding rates: the challenge of monitoring. Aust J Public Health 1994, I 8(3):337-339. 
18. Freed GL, Jones TM and Schanler RJ: Prenatal determination of demographic and attitudinal factors regarding feeding practice in an indigent population. Am J Perinatol 1992, 9(5 I6):420-424.

19. Bailey VF and Sherriff J: Reasons for the cessation of breastfeeding in women from lower socioeconomic groups in Perth Western Australia. Breastfeed Rev 1993, 8:390-393.

20. Waldenström $U$ and Nilson C-A: No effect of birth centre care on either duration or experience of breast feeding but more complications: findings from a randomised controlled trial. Midwifery 1994, 10:8-17.

21. Lawson $\mathrm{K}$ and Tulloch MI: Breastfeeding duration: prenata intentions and postnatal practices. J Adv Nurs 1995, 22:84I-849.

22. O'Campo P, Faden RR, Gielien AC and Wanc MC: Prenatal factors associated with breastfeeding duration: recommendations for prenatal interventions. Birth 1992, 19(4): I95-20I.

23. Schy DS, Maglaya CF, Mendelson SG, Race KEH and Ludwig-Beymer $P$ : The effects of in-hospital lactation education on breastfeeding practice. J Hum Lact 1996, I2(2): I I7-122.

24. Cox SG and Turnbull C): Choosing to breastfeed or bottle-feed: an analysis of factors which influence choice. Breastfeed Rev 1994, 2(1 0):459-464.

25. Minchin M: Breastfeeding Matters. Sydney. Allen \& Unwin 1989.

26. Wiles LS: The effect of prenatal breastfeeding education on breastfeeding success and maternal perception of the infant. JOGN Nurs 1984, (July/August):253-257.

27. Pugin E, Valdes V, Labbok NIH, Perez A and Aravena R: Does prenatal breastfeeding skills group education increase the effectiveness of a comprehensive breastfeeding promotion program? J Hum Lact 1996, I 2(I): 15-19.

28. Duffy E, Percival P and Kershaw E: Positive effects of an antenatal group teaching session on postnatal nipple pain, trauma and breastfeeding rates. Midwifery 1997, 13:189-196.

29. Redman S, Watkins J, Evans L and Lloyd D: Evaluation of an Australian intervention to encourage breast feeding in primiparous women. Health Promot Internation 1995, I0(2): I0I-II3.

30. Hill P: Effects of education on breastfeeding success. Matern Child Nurs J 1987, 16:145-146.

31. Jones DA and West RR: Lactation nurse increases duration of breast feeding: a randomised controlled trial. Arch Dis Child 1985, 60:772-774.

32. Frank DA, Wirtz SJ, Sorenson JR and Heeren : Commercial discharge packs and breastfeeding counselling: effects on infant feeding practices in a randomized controlled trial. Pediatrics 1987, 80:845-854.

33. McDonald SJ, Henderson JJ, Evans SF, Faulkner S and Hagan R: Effect of an extended midwifery support program on the duration of breastfeeding: a randomised controlled trial. Perinatal Society of Australia and New Zealand 7th Annual Congress 2003.

34. Fairbank L, O'Meara S, Renfrew MJ, Woolridge M, Sowden AJ and Lister-Sharp D: A systematic review to evaluate the effectiveness of interventions to promote the initiation of breastfeeding. Health Technol Assess 2000, 2(25):

35. Sikorski J and Renfrew MJ: Support for breastfeeding mothers (Cochrane Review). The Cochrane Library: Oxford 200I.

36. Ball TM and Bennett DM. The economic impact of breastfeeding. Pediatr Clin North Am 200I, 48(I):253-62.

37. Fok D, Mong TG and Chua D: The economics of breastfeeding in Singapore. Breastfeed Rev 1998, 6(2):5-9.

38. Heinig MJ: Breastfeeding and the bottom line: why are the cost savings of breastfeeding such a hard sell? J Hum Lact 1998 I 4(2):87-8.

39. Riordan JM: The cost of not breastfeeding: a commentary. J Hum Lact 1997, 13(2):93-7.

40. Montgomery DL and Splett PL: Economic benefit of breast-feeding infants enrolled in WIC. J Am Diet Assoc 1997, 97(4):379-85.

4I. Wei L L and Lachin JM: Properties of the urn randomization in clinical trials. Control Clin Trials 1988, 9(4):345-364.

42. Glover R: Attachment- the key to successful breastfeeding, in Pamphlet Australia 1994.

43. Glover R: The key to successful breastfeeding,. in Pamphlet Australia 1997.

44. Microsoft Corporation. Access 97 SR-2 1997.

45. Stata Corporation: STATA statistical data analysis. Stata Corporation: College Station, Texas 200I.
46. Begg C, Cho M, Eastwood S, Horton R, Moher D, Olkin I, Pitkin R, Rennie D, Schulz K, Simel D and Stroup D: Improving the Quality of Reporting of Randomized Controlled Trials. The CONSORT Statement. JAMA 1996, 276(8):637-639.

\section{Pre-publication history}

The pre-publication history for this paper can be accessed here:

http://www.biomedcentral.com/1471-2393/3/5/prepub
Publish with BioMed Central and every scientist can read your work free of charge

"BioMed Central will be the most significant development for disseminating the results of biomedical research in our lifetime. "

Sir Paul Nurse, Cancer Research UK

Your research papers will be:

- available free of charge to the entire biomedical community

- peer reviewed and published immediately upon acceptance

- cited in PubMed and archived on PubMed Central

- yours - you keep the copyright 